\title{
Odontomatosis-aortae esophagus stenosis syndrome
}

INSERM

\section{Source}

INSERM. (1999). Orphanet: an online rare disease and orphan drug data base.

Odontomatosis-aortae esophagus stenosis syndrome. ORPHA:2724

Odontoma-dysphagia syndrome is a malformation syndrome, characterized by odontomas (undifferentiated mass of the esophagus) and severe dysphagia. 